\title{
Identification and Expression of Acid $\beta$-Glucosidase Mutations Causing Severe Type 1 and Neurologic Type 2 Gaucher Disease in Non-Jewish Patients
}

\author{
Marie E. Grace, ${ }^{\star}$ Robert J. Desnick, ${ }^{\star \ddagger}$ and Gregory M. Pastores ${ }^{\star \ddagger}$ \\ *Department of Human Genetics, and ${ }^{\ddagger}$ Department of Pediatrics, Mount Sinai School of Medicine, New York 10029-6574
}

\begin{abstract}
Gaucher disease, the most prevalent lysosomal storage disease, occurs in three subtypes, all resulting from mutations in the acid $\beta$-glucosidase gene. Molecular studies in five severely affected type 1 and two type 2 Gaucher disease patients of non-Jewish descent identified six new mutations: K74X, W179X, G195E, S271N, V352L, and a two-base deletion in exon 10 (1450del2). Two additional mutations identified in these patients (R48W and G202R) have been reported previously, but were not expressed or characterized. Heterologous expression in Sf9 cells using the baculovirus system revealed that the missense mutations, R48W and $V 352 \mathrm{~L}$, had 14 and $7 \%$, respectively, of the specific activity based on cross-reacting immunologic material expressed by the normal allele. In contrast, the G195E, G202R, and S271N mutant alleles were more severely compromised with only 1-2\% of the normal expressed specific activity based on cross-reacting immunologic material. Structural distortion at the active site was probed by comparing the interaction of the mutant enzymes with active site-directed inhibitors (castanospermine, conduritol B epoxide and deoxynojirimycin). R48W, G202R, and S271N were normally inhibited, whereas the V352L and G195E mutant enzymes had significantly decreased binding affinity. These mutations further expand the genetic heterogeneity in the lesions causing Gaucher disease types 1 and 2, and further delineate genotype/phenotype correlations and functional domains within the acid $\beta$-glucosidase gene. (J. Clin. Invest. 1997. 99:25302537.) Key words: Baculoviridae • mutagenesis, site-directed • recombinant • enzymology • genotype
\end{abstract}

\section{Introduction}

Gaucher disease (GD), ${ }^{1}$ the most prevalent lysosomal storage disorder, results from the deficient activity of acid $\beta$-glucosi-

Address correspondence to Marie E. Grace, Ph.D., Department of Human Genetics, Mount Sinai School of Medicine, Box 1498, One Gustave Levy Place, New York, NY 10029-6574. Phone: 212-2413548; FAX: 212-360-1809; E-mail: grace@msvax.mssm.edu

Received for publication 23 January 1997 and accepted in revised form 5 March 1997.

1. Abbreviations used in this paper: 4MU-Glc, 4-methylumbelliferyl$\beta$-D-glucopyranoside; CBE, conduritol B epoxide; CRIM, crossreacting immunologic material; CRIM SA, CRIM specific activity; CS, castanospermine; DNM, deoxynojirimycin; GD, Gaucher disease; Hgb, hemoglobin; NBD-GC, NBD-glucosylceramide.

J. Clin. Invest.

(C) The American Society for Clinical Investigation, Inc. 0021-9738/97/05/2530/08 \$2.00

Volume 99, Number 10, May 1997, 2530-2537 dase, and the accumulation of glucosylceramide in monocytes/ macrophages $(1,2)$. The disease is an autosomal recessive disorder $(1,2)$ resulting from mutations in the acid $\beta$-glucosidase (EC.3.2.1.45) gene, which has been localized to the chromosomal region 1q21-q31 (3-5). Three clinical subtypes have been delineated based on the absence (type 1) or presence and severity (acute type 2 and subacute type 3 ) of neurological involvement. Although prevalent among individuals of Ashkenazi Jewish descent, the disease is panethnic. Almost 80 gene alterations leading to the defective activity of this lysosomal hydrolase have been identified (for review see Beutler and Gelbart, reference 6). Four defects (N370S, 84insG, L444P, and IVS $2^{+1}$ ), account for $90-96 \%$ of the disease-causing changes among patients of Ashkenazi descent $(7,8)$. In nonJewish patients, the N370S and L444P mutations account for 38 and $33 \%$ of disease alleles, respectively (9). Of the remaining mutations reported, most are unique or "private," having been detected in only one or a few GD families.

The full length cDNA and genomic sequences for acid $\beta$-glucosidase have been isolated and characterized (10-12). Of note, a pseudogene transcribable into mRNA, but unable to produce a functional gene product (13), is located $16 \mathrm{~kb}$ downstream from the structural gene (12), and several mutations causing GD derived from homologous recombination and/or gene conversion have been reported. The structural gene codes for a 497 amino acid mature protein of $59-67 \mathrm{kD}$, depending on the state of glycosidic processing. Acid $\beta$-glucosidase is active as a monomer and requires saposin $\mathrm{C}$, a small glycoprotein activator, for physiological activity (14). Detailed characterization studies of heterologously expressed normal and mutagenized acid $\beta$-glucosidases have localized residues important for substrate and activator binding to the last third of the protein (15-17) and identified $\mathrm{Glu}^{340}$ as the catalytic nucleophile (18).

The identification and expression of mutations in the acid $\beta$-glucosidase gene is useful for structure/function studies and for genotype/phenotype correlations. In this communication, the identification and characterization of six new and two previously uncharacterized mutations in five moderately to severely affected type 1 patients and two severely affected type 2 patients, all of non-Jewish ancestry, are described. Heterologous expression of the missense mutations was undertaken to characterize the mutant enzymes' stability and catalytic function, confirming causality for GD. An additional mutation, the substitution of the glutamate at position 235 by glycine (E235G), was engineered to probe this residue's putative mechanistic role as the acid catalyst (19). These studies led to further delineation of functional regions and identification of individual residues important for maintaining normal acid $\beta$-glucosidase function.

\section{Methods}

Patient descriptions. Patient 1 is a 20 -yr-old female of Indian descent with severe type 1 GD who presented with cachexia and massive 
hepatosplenomegaly (liver and spleen volumes of 3,095 and 6,545 ml, respectively), ascites, and portal hypertension. She had marked anemia (hemoglobin [Hgb], $9.2 \mathrm{~g} \%$ ) and thrombocytopenia $(28,000 /$ $\mathrm{mm}^{3}$ ). The diagnosis was confirmed by demonstration of markedly deficient leukocyte acid $\beta$-glucosidase activity $(0.8 \mathrm{nmol} / \mathrm{h}$ per $\mathrm{mg}$; normal range $6.5 \pm 0.5 \mathrm{nmol} / \mathrm{h}$ per $\mathrm{mg}$ ). The parents were nonconsanguineous, and a family history revealed that a similarly affected younger male sibling had died at age 8 , presumably due to complications of severe type $1 \mathrm{GD}$.

Patient 2 is a 3.5-yr-old African-American male with severe type $1 \mathrm{GD}$. He presented at $1.5 \mathrm{yr}$ of age with anemia ( $\mathrm{Hgb}, 9.6 \mathrm{~g} \%)$ and hepatosplenomegaly (liver and spleen volumes of 704 and $290 \mathrm{ml}$, respectively). Bone marrow examination revealed Gaucher cells, and patient had a markedly diminished leukocyte acid $\beta$-glucosidase activity $(0.6 \mathrm{nmol} / \mathrm{h}$ per $\mathrm{mg})$. The child's parents were not related.

Patient 3 is a 30 -yr-old female of English descent with severe type 1 GD who was diagnosed at age 6 mo when she presented with splenomegaly. Before therapy at age 27 , she had undergone a splenectomy and suffered from severe bone involvement with pathological pelvic fracture during a pregnancy at age 26. She had anemia (Hgb, $9.7 \mathrm{~g} \%$ ), hepatomegaly (three times normal liver volume), and diminished leukocyte acid $\beta$-glucosidase activity $(1.0 \mathrm{nmol} / \mathrm{h}$ per $\mathrm{mg})$.

Patient 4 is a 31-yr-old female of Hispanic (Peruvian) descent with moderate type 1 GD. She presented with easy bruising and bone pain and was diagnosed by bone marrow biopsy at age 8 . At age 20, she had a partial splenectomy. At age 27 , she had markedly diminished leukocyte acid $\beta$-glucosidase activity $(0.8 \mathrm{nmol} / \mathrm{h}$ per $\mathrm{mg})$, anemia (Hgb, $6.0 \mathrm{~g} \%$ ), thrombocytopenia (platelet count $80,000 / \mathrm{mm}^{3}$ ), hepatosplenomegaly (with liver and spleen volumes 2.7 and 18 times normal, respectively), and bone disease characterized by lytic femoral lesions.

Patient 5 is an 18-yr-old female of Hispanic (Spanish) descent with moderate type 1 GD who was diagnosed at age 16 by bone marrow because of anemia ( $\mathrm{Hgb}, 10.3 \mathrm{~g} \%$ ), mild splenomegaly (six times normal spleen volume), and diminished leukocyte acid $\beta$-glucosidase activity $(0.7 \mathrm{nmol} / \mathrm{h}$ per $\mathrm{mg})$. She experienced extreme fatigue before enzyme therapy that has resolved together with significant improvement in her hemoglobin and normalization of her spleen volume.

Patient 6 is a 2-yr-old Hispanic male from Puerto Rico with type 2 GD. He presented with developmental delay, strabismus, and hepatosplenomegaly (liver and spleen volumes of 773 and $377 \mathrm{ml}$, respectively) at age $7 \mathrm{mo}$. A metabolic workup revealed markedly diminished acid $\beta$-glucosidase activity. The patient developed severe myoclonic seizures that were poorly controlled by anticonvulsant medications and continued to degenerate neurologically.

Patient 7 was a male child of Italian descent with type 2 GD who presented with hepatosplenomegaly at age 4 mo and died secondary to neurologic involvement at age $1.5 \mathrm{yr}$. His parents were first cousins. A postmortem examination was not performed and no other clinical information is available.

Reagents. Triton X-100 and NBD-glucocerebroside were obtained from Sigma Chemical Co. (St. Louis, MO). Ethylenediamine was purchased from Fisher Scientific Co. (Pittsburgh, PA). 4-methylumbelliferyl- $\beta$-D-glucopyranoside and recombinant $\mathrm{N}$-glycanase ${ }^{\mathrm{TM}}$ were from Genzyme, Corp. (Cambridge, MA). Sodium taurocholate, de- oxynojirimycin, and castanospermine were purchased from Calbiochem Corp. (La Jolla, CA). Dynabeads ${ }^{\circledR}$ were obtained from DYNAL, Inc. (Great Neck, NY). The Sculptor ${ }^{\mathrm{TM}}$ In Vitro Mutagenesis System kit was from Amersham Corp. (Arlington Heights, IL). Spodoptera frugiperda (Crl 1711) cells were purchased from American Type Culture Collection (Rockville, MD). Baculogold ${ }^{\circledR}$ was obtained from PharMingen (San Diego, CA). 2,4-dinitrophenol- $\beta$-glucopyranoside was a kind gift of Stephen G. Withers (University of British Columbia, Vancouver, B.C., Canada). Instruments used were: a Cell Disruptor 200 (Branson Ultrasonics Corp., Danbury, CT), a fluorometer (450; Sequoia-Turner Corp., Mountain View, CA), and an Optical System 3 spectrofluorometer (Farrand, New York).

DNA isolation, PCR amplification, and mutation detection. Genomic DNA was isolated from peripheral blood leukocytes using standard techniques (20). Each sample was initially screened for the four common mutations (N370S, L444P, 84insG, and IVS2 ${ }^{+1}$ ) by PCR amplification and restriction digestion (21-24). To detect the unknown acid $\beta$-glucosidase mutations, the complete coding region and adjacent intron/exon boundaries were amplified from genomic DNA (500 ng) using biotinylated sense (exons $1+2,3,4,6$, and 7) and antisense (exons 5, $8+9$, and $10+11$ ) primers as previously described (23). Table I lists the primers used for specific amplification of the exons of the structural gene in which novel mutations were found. The relevant PCR product was denatured with $\mathrm{NaOH}$, and the biotinylated single stranded template was subsequently immobilized on streptavidin attached to polystyrene Dynabeads ${ }^{\circledR}$. Solid-phase direct sequencing was performed by the dideoxy chain termination method $(25,26)$.

The designations for the mutations refer to the position of the amino acid substitution, where amino acid 1 is the $\mathrm{NH}_{2}$ terminus of the mature protein. The cDNA base numbers refer to the position of the nucleotide in the cDNA $(10,27)$, where nucleotide 1 is the A in the first ATG. The genomic designations are based on the updated acid $\beta$-glucosidase Genbank/EMBL/DDBJ sequence (accession number J03059; 8/95)(12, 28), from which the first 874 nucleotides in the 5 '-untranslated region have been deleted. The first nucleotide of exon 1 is at genomic position 356.

Construction of expression plasmids. Point mutations for R48W, G195E, G202R, S271N, V352L, and E235G were introduced into the acid $\beta$-glucosidase cDNA by an M13mp19-based oligonucleotidedirected site-specific mutagenesis procedure (Sculptor ${ }^{\mathrm{TM}}$ In Vitro $\mathrm{Mu}-$ tagenesis System) employing the phosphorothioate selection method $(29,30)$, as previously described (17). The complete sequence of each mutagenized cDNA was determined to confirm that no spurious mutations were incorporated during the mutagenesis procedure. The mutant cDNAs were then cloned into the EcoRI site of the baculovirus expression vector, $p A c 1392$. The final acid $\beta$-glucosidase cDNA inserts in the expression plasmid comprised a 1,562-bp fragment beginning 12 nucleotides upstream of the second ATG, ending at the stop codon, and containing the correct amino acid (arginine) at position 495 .

Construction and purification of recombinant baculovirus. Recombinant baculovirus containing each of the different acid $\beta$-glucosidase cDNAs (normal in the sense [NL] and antisense [Rev] directions, R48W, G195E, G202R, S271N, V352L, and E235G) were

Table I. Oligonucleotide Primers Used to Amplify Exons 3, 4, 6, 7, 8, and 10 of the Acid $\beta$-Glucosidase Structural Gene

\begin{tabular}{|c|c|c|}
\hline Exon & Sense primers & Antisense primers \\
\hline 3 & 5' - CATGTCTTCATCAGACCTCAC - 3' & 5' - CACAGGGAATAAGGGTATCAG - 3' \\
\hline 4 & 5' - GCTGGGTACTGATACCCTTAT - 3' & 5' - TGGGCAGAGTGAGATTCTG - 3' \\
\hline 6 & 5'-GCTTCTCTCTTCACTACCTT-3' & 5' - GGTGACAGAGAGAGAGACTCC - 3' \\
\hline 7 & 5' - GGTCTGGTCCACTTTCTTGG - 3' & 5' - GTTTCAAGCGACAACTGTGG - 3' \\
\hline 8 & 5' - AGGCTAATGTGGGAGGATC - 3' & 5' - GTAGGAGATGATAGGCCTGGTA - 3' \\
\hline 10 & 5' - CGTAACTTTGTCGACAGT - 3' & 5' - ATAAAGCCACTCACACTGA - 3' \\
\hline
\end{tabular}


produced in cloned $S$. frugiperda (Sf9) cells by calcium phosphatemediated transfection and homologous recombination between the expression plasmid and baculovirus genomic DNA (Baculogold ${ }^{\circledR}$ ) as described (31). Pure recombinant baculovirus clones containing the normal or mutant cDNA for acid $\beta$-glucosidase were isolated by plaque hybridization, amplified, titered, and used at a multiplicity of infection $>10$ to infect $\mathrm{Sf} 9$ cells (31).

Immunoblotting. Immunoelectroblotting using a polyclonal antihuman acid $\beta$-glucosidase antibody was conducted as described (15-17). In brief, Sf9 cells infected with pure recombinant virus were harvested $3 \mathrm{~d}$ after infection. The pellets were sonicated in $0.04 \mathrm{M}$ citrate/phosphate buffer, pH 5.5, containing $1 \mathrm{mM}$ EDTA, $4 \mathrm{mM} \beta$-mercaptoethanol, $0.1 \%$ Triton X-100, and $0.1 \%$ sodium taurocholate, using a cup sonicator (Cell Disruptor 200; 80 watts, pulse times of 30, 20 , and $20 \mathrm{~s})$. Aliquots of the clarified $(875 \mathrm{~g}, 20 \mathrm{~min})$ crude sonicates containing determined amounts of protein and enzymatic activity were run on tricine-SDS-polyacrylamide gels (32) and immunoblotted. Specific activities based on the amount of cross-reacting immunological material (CRIM) specific activity (CRIM SA) were determined as described $(15,16)$. The relative amounts of CRIM per unit of glucosylceramide-cleaving activity for each mutant was determined by visual inspection of immunoblotting membranes and referenced to that of the normal enzyme.

Enzyme assays. Aliquots of the crude sonicates, prepared as described above, were assayed for acid $\beta$-glucosidase activity using the fluorescently labeled natural substrate (NBD-glucopyranosylceramide or 12-[ $N$-methyl- $N$-(7-nitrobenz-2-oxa-1,3-diazol-4-yl)]dodecanoyl-sphingosyl-1-O- $\beta$-D-glucopyranoside; NBD-GC) or the synthetic substrate (4-methylumbelliferyl- $\beta$-D-glucopyranoside; 4MU-Glc). The final reaction mixtures contained either $0.3 \mathrm{mM}$ NBD-GC (100 $\mu \mathrm{l}$ final reaction vol) or $4 \mathrm{mM} 4 \mathrm{MU}-G \mathrm{cc}(200 \mu \mathrm{l}$ final reaction vol $)$ in $0.04 \mathrm{M}$ citrate/phosphate buffer, $\mathrm{pH} 5.5,1 \mathrm{mM}$ EDTA, $4 \mathrm{mM} \beta$-mercaptoethanol, $0.25 \%$ Triton $\mathrm{X}-100$, and $0.25 \%$ sodium taurocholate. The 4MU-Glc reaction was terminated by raising the $\mathrm{pH}$ with the addition of $2.3 \mathrm{ml}$ of a $0.1 \mathrm{M}$ aqueous solution of ethylenediamine. The fluorescence of the samples were then read with the fluorometer. The NBD-GC assays were terminated and processed as described (33). The fluorescence of the extracted reaction products were read using an Optical System 3 spectrofluorometer. Background levels were determined by comparison with results obtained from Sf9 cells infected with recombinant baculovirus containing the cDNA for acid $\beta$-glucosidase in the antisense direction. $1 \mathrm{U}$ acid $\beta$-glucosidase activity is the amount of enzyme that hydrolyzed $1 \mu \mathrm{M}$ substrate $/ \mathrm{min}$ at $37^{\circ} \mathrm{C}$.

The effect of the addition of sodium azide on the activity of the E235G mutant was measured using the stopped assays outlined above with the natural (NBD-GC) and synthetic (4MU-Glc) substrates and a continuous assay with 2,4-dinitrophenol- $\beta$-glucopyranoside (DNP-Glu) as the substrate. Initial rates of hydrolysis towards both NBD-GC and 4MU-Glc (100 and $200 \mu$ l final reaction vol, respectively) were determined in the presence of $0,10,250$, and 500 $\mathrm{mM}$ sodium azide by the addition of aliquots from a concentrated (4.8 M) aqueous sodium azide solution just before addition of crude E235G lysate. For the continuous assay, the reaction mixture containing $0.39 \mathrm{mM}$ DNP-Glu in $0.04 \mathrm{M}$ citrate/phosphate buffer, $\mathrm{pH} 5.5$, $0.25 \%$ Triton X-100, and $0.25 \%$ sodium taurocholate with or without $200 \mathrm{mM}$ sodium azide was preincubated at $37^{\circ} \mathrm{C}$ in a thermostatted cell holder. An aliquot of E235G lysate was added and the release of dinitrophenolate was followed continuously by measuring the increase in absorbance at $400 \mathrm{~nm}$ at 10 -s intervals.

Inhibition of expressed normal and mutant acid $\beta$-glucosidases. Aliquots of crude lysates were assayed in the absence or presence of each of the following active site-directed inhibitors: conduritol $\mathrm{B}$ epoxide (CBE; 0-600 $\mu \mathrm{M})$, deoxynojirimycin (DNM; 0-600 $\mu \mathrm{M})$, and castanospermine (CS; 0-80 $\mu \mathrm{M})$. Concentrated aqueous stock solutions of the inhibitors were made such that aliquots between 0 and 50 $\mu l$ gave the desired final concentrations in the reaction assay. The synthetic substrate (4MU-Glc) was used. The final reaction mixture contained $4 \mathrm{mM} 4 \mathrm{MU}-\mathrm{Glc}(200 \mu \mathrm{l}$ final reaction vol $)$ and the appro- priate amount of inhibitor in $0.04 \mathrm{M}$ citrate/phosphate buffer, $\mathrm{pH} 5.5$, containing $0.25 \%$ Triton $\mathrm{X}-100$ and $0.25 \%$ sodium taurocholate. The $\mathrm{IC}_{50}$ for each inhibitor for the normal and mutant (R48W, G195E, G202R, S271N, and V352L) acid $\beta$-glucosidases was determined from a plot of activity remaining versus inhibitor concentration. The previously characterized (15-17) common mutations, N370S and L444P, were reexpressed for comparative purposes.

$N$-Glycanase treatment of expressed normal and mutant acid $\beta$-glucosidases. Sf 9 cells expressing normal acid $\beta$-glucosidase and two of the mutants, R48W and V352L, were harvested and sonicated in $0.2 \mathrm{M}$ phosphate buffer, $\mathrm{pH} 7.5,(200 \mu \mathrm{l})$ as described above. Aliquots of the crude lysates $(100 \mu \mathrm{l})$ were adjusted to $0.5 \%$ SDS and $0.1 \mathrm{M} \beta$-mercaptoethanol and boiled for $5 \mathrm{~min}$. Octyl- $\beta$-glucopyranoside $(10 \%$ stock solution) was added to a final concentration of $2.6 \%$ (i.e., a sevenfold excess over the final SDS concentration of $0.37 \%$ ). Each sample was divided in half. Recombinant $N$-Glycanase ${ }^{\circledR}(0.3 \mathrm{U})$ was added to one aliquot of each sample, while the other served as the untreated control. After $8 \mathrm{~h}$, the deglycosylation reaction was terminated by addition of the protein gel loading dye. The samples were analyzed by tricine-SDS-PAGE (10\% acrylamide gel) and Western blotting, as previously described $(16,17)$.

\section{Results}

Identification of novel point mutations. Complete sequencing of the acid $\beta$-glucosidase coding region and intron/exon boundaries from seven non-Jewish patients with GD (Table II) resulted in the identification of five new point mutations (K74X, W179X, G195E, S271N, and V352L), and a novel 2-bp deletion in exon 10 (1450del2), as well as two known but uncharacterized mutations (R48W and G202R) (34, 35). Other alleles found included the common N370S, the complex RecNciI, and the rare but previously expressed F213I $(22,36)$ lesions. Table III summarizes the identified nucleotide substitutions and the resulting changes at the protein level.

Six of the novel mutations were found in five patients with nonneurologic disease. Patient 1 , with severe GD type 1 , was heteroallelic for R48W and W179X. Although the nucleotide substitution at position 259 in the cDNA causing the R48W mutation corresponds to the pseudogene sequence at the analogous site, it did not derive from a crossover event between the structural gene and the pseudogene since other pseudogenelike substitutions at contiguous sites were not present (nucleotides 257-261 for the normal allele, GACGG; and the R48W allele, GATGG; corresponding to pseudogene positions ATTGG). Moreover, it occurred at a CpG dinucleotide, a known mutational hotspot $(37,38)$. Patient 2 , with severe type 1 disease, did not carry a normal V352 codon and appeared homoallelic for V352L (Fig. $1 A$ ). The genomic DNAs

Table II. Phenotypes, Ancestry, and Genotypes of the Non-Jewish Patients with Gaucher Disease

\begin{tabular}{llll}
\hline Patient & \multicolumn{1}{c}{ Phenotype } & \multicolumn{1}{c}{ Ancestry } & \multicolumn{1}{c}{ Genotype } \\
\hline 1 & Type 1, severe & Indian & R48W/W179X \\
2 & Type 1, severe & African-American & V352L/del \\
3 & Type 1, severe & English & S271N/RecNciI \\
4 & Type 1, moderate & Hispanic (Peruvian) & G195E/N370S \\
5 & Type 1, moderate & Hispanic (Spanish) & 1450del2/N370S \\
6 & Type 2 & Hispanic (Puerto Rican) & K74X/F213I \\
7 & Type 2 & Italian & G202R/G202R
\end{tabular}


Table III. Mutations Identified in Non-Jewish Patients with Gaucher Disease

\begin{tabular}{|c|c|c|c|c|c|}
\hline Designation & cDNA position & Genomic position & Codon change & Exon & Amino acid change \\
\hline R48W & 259 & 1763 & $\underline{\mathrm{CGG}} \rightarrow \underline{\mathrm{TGG}}$ & 3 & Arginine $\mathrm{A}^{43} \rightarrow$ tryptophan \\
\hline K74X & 337 & 1964 & $\underline{\overline{\mathrm{A}} \mathrm{AG}} \rightarrow \underline{\overline{\mathrm{T}}} \mathrm{AG}$ & 4 & Lysine ${ }^{74} \rightarrow$ termination \\
\hline W179X & 653 & 3455 & $\overline{\mathrm{T}} \underline{\mathrm{GG}} \rightarrow \overline{\mathrm{T}} \underline{\mathrm{A} G}$ & 6 & Tryptophan ${ }^{179} \rightarrow$ termination \\
\hline G195E & 701 & 3503 & $\overline{\mathrm{GG}} \underline{\underline{G}} \rightarrow \overline{\mathrm{GA}} \mathrm{G}$ & 6 & Glycine $^{195} \rightarrow$ glutamate \\
\hline G202R & 721 & 3523 & $\underline{\mathrm{G}} \overline{\mathrm{G}} \mathrm{A} \rightarrow \mathrm{A} \overline{\mathrm{G}} \mathrm{A}$ & 6 & Glycine $^{202} \rightarrow$ arginine \\
\hline S271N & 929 & 4286 & $\overline{\mathrm{A}} \underline{\mathrm{GT}} \rightarrow \overline{\mathrm{A}} \underline{\mathrm{AT}}$ & 7 & Serine $e^{271} \rightarrow$ asparagine \\
\hline V352L & 1171 & 5399 & $\underline{\mathrm{GTG}} \rightarrow \underline{\mathrm{CT} G}$ & 8 & Valine ${ }^{352} \rightarrow$ leucine \\
\hline 1450del2 & $1451-1452$ & $6448-6449$ & $\overline{\mathrm{de}} \mathrm{A}+\overline{\mathrm{C}}$ & 10 & $\begin{array}{r}\ldots \text { - } \text { L }^{444} \text {-D-A-V-A-L-M-H-P- } \ldots \text { - }-\mathrm{Q}^{497} \rightarrow \\
\ldots \text { - } \text { L }^{444} \text {-G-S-G-T-D-G-S-R }{ }^{452} \text {-term }\end{array}$ \\
\hline
\end{tabular}

from both parents were amplified across exon 8 and sequenced. The mother was heteroallelic for the V352L mutation, while codon 352 in the father was normal, suggesting that both the father and the son had an unamplifiable allele (i.e., deletion). Efforts to define the limits of the deletion are underway. Patient 3 , with moderate to severe type 1 disease, was heteroallelic for the S271N mutation and the common complex allele, RecNciI (L444P+A456P+V460V). Patient 4, with moderate type 1 disease, was heteroallelic for G195E and N370S, the common exon 9 defect. Patient 5, with moderate to severe type 1 disease, was heteroallelic for a two-base deletion after nucleotide 1450 in exon 10 (1450del2) (Fig. $1 B$ ) and the common N370S allele. The deletion of the two bases at nucleotide positions 1451 and 1452 in codon 445 causes a shift in reading frame and the translation of eight altered bases after Leu $^{444}$ (Gly ${ }^{445}$-Ser-Gly-Thr-Asp-Gly-Ser-Arg ${ }^{452}$ ) before reaching a premature termination codon after position 452 . The deleted area includes residues required for substrate and activator binding (17).

The remaining two mutations were identified in two patients with the most severe neurologic form of the disease, type 2. Patient 6 was heteroallelic for K74X and F213I. The F213I was reported previously in two Japanese type 2 patients, one Japanese type 3 patient, and a patient of Hungarian/German descent with severe GD type $1(22,36)$. Although no samples were available from patient 7 , who died at $1.5 \mathrm{yr}$ from complications of GD type 2, both parents, who were first cousins, carried the same exon 6 mutation, G202R. This substitution mimics the base substitution in the pseudogene at the analogous site.

The K74X and W179X alleles are predicted to produce unstable protein products that terminate before all the active site residues identified to date (17). The 452 amino acid truncated protein expressed from the $1450 \mathrm{del} 2$ allele would be missing residues important for completing the active site. These three severely compromised alleles were not characterized further.

Expression and characterization of missense mutations. The R48W, G195E, G202R, S271N, and V352L missense alleles, as well as the sense and antisense acid $\beta$-glucosidase alleles, were expressed in the baculovirus system and characterized (Table IV). The previously studied N370S and L444P alleles $(15,17)$ were reexpressed for comparative purposes. In addition, the mutation, E235G, which was created by the substitution of an adenine at position 821 in the cDNA (genomic position 4178 ) by guanine $(\mathrm{GAG} \rightarrow \mathrm{GGG})$, leading to the replacement of the glutamate at residue 235 by glycine, was also included in these studies to probe the putative role of $\mathrm{Glu}^{235}$ as the acid catalyst (19). Using the fluorescently labeled natural substrate, NBD-GC, the normal sense construct expressed $\sim 4.0 \times 10^{-2} \mu \mathrm{mol} / \mathrm{min}$ of glucosylceramide-cleaving activity per milligram of total protein. Essentially no activity towards the natural substrate, glucosylceramide, was detected in Sf9 cells infected with recombinant baculovirus containing the acid $\beta$-glucosidase cDNA in the antisense orientation. All expressed missense alleles had significantly reduced activity in their crude lysates. The R48W lysates had the greatest residual activity ( $\sim 28 \%$ with respect to the normal), with levels similar to those from the expressed mild N370S allele. V352L had the second highest activity, with slightly $>11 \%$ of the normal residual activity. The remaining patient-derived mutants, G195E, G202R, and S271N, had $<5 \%$ residual activity. The E235G mutant was the most severely compromised, with crude lysates having $<0.5 \%$ the residual activity of the normal.

As shown in Fig. 2, lanes 2 and 9, the normal cDNA for human acid $\beta$-glucosidase expressed in Sf9 cells produced a multiband pattern that reacted strongly with polyclonal anti-
A

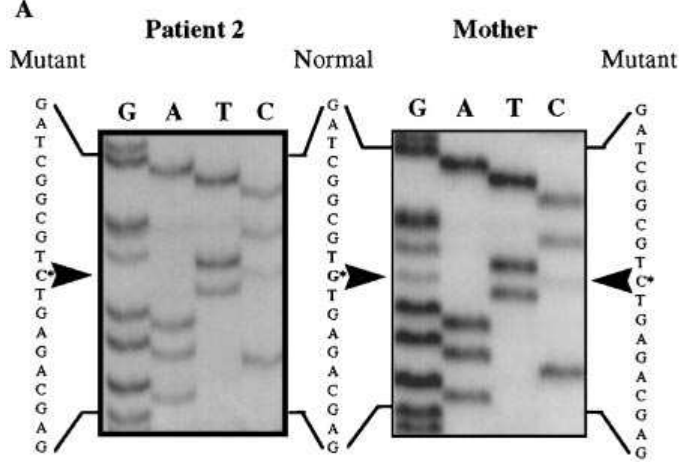

B

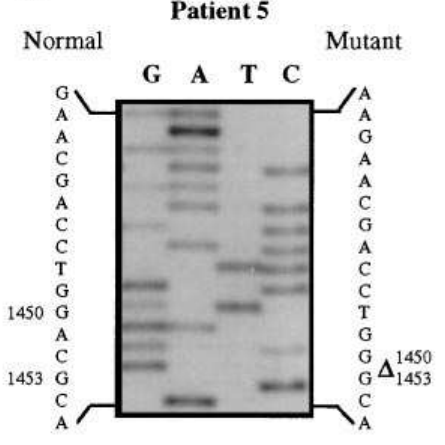

Figure 1. Single-stranded sequence analysis of the biotinylated strand after PCR-amplification of $55 \mathrm{ng}$ of genomic DNA. (A) Amplification of exon 8 in patient 2 and one parent illustrating the V352L mutation. (B) Amplification of exon 10 of patient 5 depicting the heterozygous presence of the $1450 \mathrm{del} 2$ mutation. 
Table IV. Characterization of Acid $\beta$-Glucosidases Expressed in Sf9 Cells

\begin{tabular}{lccccc}
\hline & & & \multicolumn{3}{c}{$\mathrm{IC}_{50} \mathrm{~S}^{\S}$} \\
\cline { 4 - 6 } cDNA & Specific activity* & CRIM SA $^{\ddagger}$ & $\mathrm{CS} \|$ & $\mathrm{DMN}^{\|}$ & $\mathrm{CBE}^{\|}$ \\
\hline & $\mu$ mol GC/min per mg & & $\mu M$ & $\mu M$ & $\mu M$ \\
Normal & $4.0 \times 10^{-2}$ & 1.0 & $9-13$ & $88-125$ & $23-47$ \\
Antisense & $0.0^{\text {q }}$ & - & - & - & - \\
R48W & $1.1 \times 10^{-2}$ & 0.14 & 13 & 150 & 39 \\
G195E & $1.5 \times 10^{-3}$ & 0.01 & $30-80$ & $385-600$ & $335-340$ \\
G202R & $1.6 \times 10^{-3}$ & 0.01 & $10-16$ & $125-160$ & $47-59$ \\
S271N & $1.4 \times 10^{-3}$ & 0.02 & $9-11$ & $96-140$ & $35-51$ \\
V352L & $4.6 \times 10^{-3}$ & 0.07 & 25 & 300 & 56 \\
N370S & $1.0 \times 10^{-2}$ & $0.13^{* *}$ & $16-25$ & $520-540$ & $88-180$ \\
L444P & $1.9 \times 10^{-3}$ & $0.05 * *$ & $9-14$ & $129-135$ & $47-55$ \\
E235G & $1.7 \times 10^{-4}$ & 0.002 & - & - & - \\
& & & & & \\
\hline
\end{tabular}

*Using NBD-labeled glucosylceramide as substrate. ${ }^{*}$ CRIM SA, total units of specific activity per cross-reacting immunological material. ${ }^{8} \mathrm{IC}_{50} \mathrm{~s}$, concentration of inhibitor resulting in $50 \%$ loss of activity towards 4MU-Glc. "Inhibitors: CS, castanospermine; DNM, deoxynojirimycin; $\mathrm{CBE}$, conduritol $\mathrm{B}$ epoxide. "There is no CBE-inhibitable activity in crude lysates from cells expressing the antisense cDNA. **CRIM SA values from previous mutagenesis studies in this laboratory (17).

human acid $\beta$-glucosidase antibody. The smallest band $\left(M_{\mathrm{r}}\right.$ $\sim 56,000)$ can be clearly seen on the immunoblot from the more heavily loaded gel (Fig. 2, lane 9). Similar patterns were obtained for all of the expressed mutant cDNAs. No CRIM was detected in the cells expressing the antisense construct. The origin of the multiband pattern was explored using the expressed normal enzyme and the two mutants, R48W and V352L. The bands $\left(M_{\mathrm{r}}\right.$ ranging from 63,000 to $\left.\sim 56,000\right)$ represented differentially glycosylated forms of acid $\beta$-glucosidase, since after treatment with N-Glycanase ${ }^{\circledR}$ (Fig. 3), a single species of $\sim 55,600 \mathrm{D}$ was observed, consistent with that of the deglycosylated mature acid $\beta$-glucosidase protein (1). Interestingly, the migration pattern of the lowest band in the untreated normal and mutant samples remained unchanged after N-Glycanase ${ }^{\circledR}$ treatment. The molecular weight of this lower band is identical to the mature deglycosylated protein, indicating that

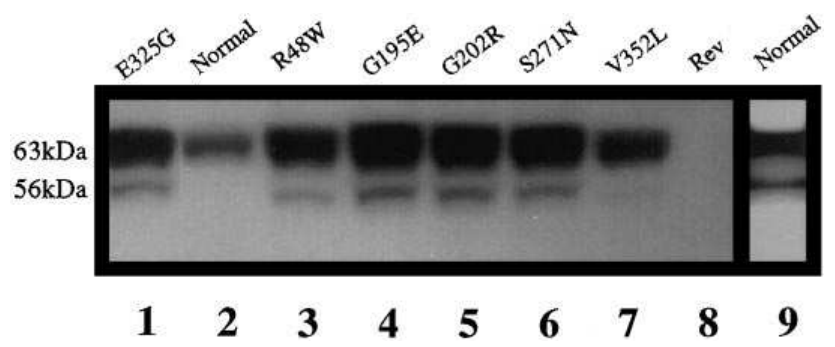

Figure 2. Composite immunoblot of the normal and mutant acid $\beta$-glucosidases expressed in Sf9 cells. Aliquots of crude lysates from gel 1 (lanes 1-8) containing E235G $\left(1.3 \times 10^{-5} \mathrm{U}, 80 \mu \mathrm{g}\right)$, normal $\left(3.7 \times 10^{-3} \mathrm{U}, 92 \mu \mathrm{g}\right), \mathrm{R} 48 \mathrm{~W}\left(1.5 \times 10^{-3} \mathrm{U}, 136 \mu \mathrm{g}\right), \mathrm{G} 195 \mathrm{E}(1.5 \times$ $\left.10^{-4} \mathrm{U}, 102 \mu \mathrm{g}\right), \mathrm{G} 202 \mathrm{R}\left(1.3 \times 10^{-4} \mathrm{U}, 83 \mu \mathrm{g}\right), \mathrm{S} 271 \mathrm{~N}\left(1.4 \times 10^{-4} \mathrm{U}, 100\right.$ $\mu \mathrm{g}), \mathrm{V} 352 \mathrm{~L}\left(4.7 \times 10^{-4} \mathrm{U}, 103 \mu \mathrm{g}\right)$, and the antisense construct (Rev, $103 \mu \mathrm{g}$ ); and gel 2 (lane 9) containing normal $\left(8.0 \times 10^{-3} \mathrm{U}, 200 \mu \mathrm{g}\right)$ were analyzed by tricine-SDS-PAGE $(10 \%)$, followed by immunoblotting using a polyclonal anti-human acid $\beta$-glucosidase antibody.

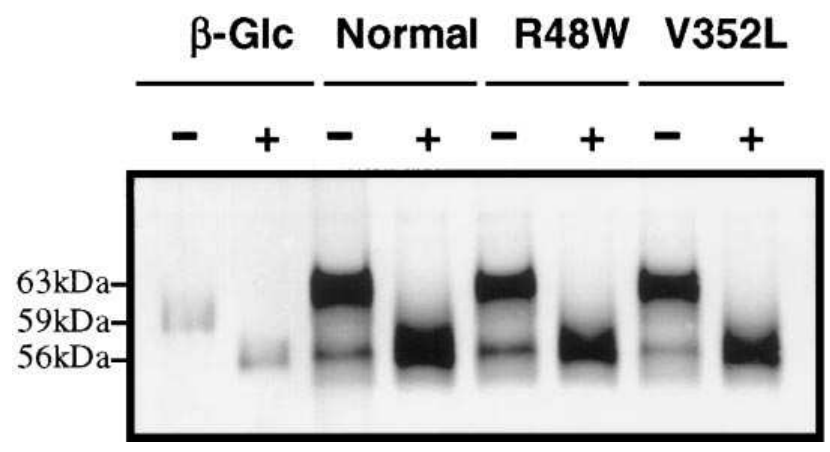

Figure 3. N-Glycanase treatment of expressed normal, R48W, and V352L acid $\beta$-glucosidases from Sf9 cells. Aliquots of crude sonicates were incubated in the absence $(-)$ and presence $(+)$ of N-Glycanase $^{\circledR}$. The samples were analyzed by tricine-SDS-PAGE $(10 \%)$, followed by immunoblotting using a polyclonal anti-human acid $\beta$-glucosidase antibody.

it has undergone appropriate signal peptide cleavage in the endoplasmic reticulum, but no cotranslational glycosidic processing. Presumably, the high levels of protein synthesis achieved in Sf9 cells with the baculovirus expression vectors overwhelmed the glycosylation machinery in the ER, resulting in unglycosylated and underglycosylated species.

All sense cDNAs gave strong CRIM signals (Fig. 2), indicating similar stabilities for the normal and mutant enzyme species. To assess the level of catalytic efficiency $\left(k_{\text {cat }}\right)$ for each mutant allele, the amount of CRIM SA was calculated for each relative to the expressed normal acid $\beta$-glucosidase (Table IV). In comparison with the signal from the expressed normal enzyme in the immunoblot in Fig. 2, the lane containing R48W had approximately three times the CRIM (i.e., three times the amount of acid $\beta$-glucosidase protein). Since only $41 \%$ of the normal activity was loaded, the R48W enzyme had a 7.3-fold reduction in CRIM SA or catalytic efficiency compared with the expressed normal enzyme. Similarly, comparison of the CRIM signals and activities for the normal and V352L proteins indicated a 15-fold reduction in catalytic efficiency for the V352L-expressed enzyme. The effects of the remaining mutations were much more deleterious, all causing an $\sim 100$-fold (G195E, G202R, and S271N) or greater (E235G) reduction in turnover rate or $k_{\text {cat }}$.

To probe the effect of the various amino acid substitutions on the structural integrity of the active site of each mutant protein species, the affinity of various active site-directed inhibitors (DNM, CBE, and CS) were assessed by determination of the concentration of each inhibitor required for inhibition of $50 \%$ of the initial activity $\left(\mathrm{IC}_{50} \mathrm{~s}\right)$. Three of the mutants, $\mathrm{R} 48 \mathrm{~W}$, G202R, and S271N, interacted normally with the series of inhibitors tested (as did L444P) and had $\mathrm{IC}_{50}$ values that overlapped with those for the expressed normal enzyme. As was the case with N370S, the mutants, G195E and V352L, demonstrated significantly reduced affinity for DNM and CBE. Interestingly, the range for the $\mathrm{IC}_{50}$ values for CS towards the G195E mutant enzyme was considerably higher than any mutant characterized to date (15-17). The extremely low residual activity of the E235G mutant precluded its inclusion in the inhibition studies. This mutant was, however, tested for a gain of activity in the presence of a strong competitive nucleophile, sodium azide. This criterion has been used to identify the acid catalyst 
in Agrobacterium faecalis $\beta$-glucosidase (39). There was no change in activity of E235G towards glucosylceramide or $4 \mathrm{MU}-\mathrm{Glc}$ in the presence of up to $250 \mathrm{mM}$ sodium azide or towards 2,4-dinitrophenol- $\beta$-glucopyranoside in the presence of $200 \mathrm{mM}$ sodium azide.

\section{Discussion}

The identification of these mutations and their individual expression in the baculovirus system provided insight into the molecular basis of the GD subtypes and structure/function relationships in normal acid $\beta$-glucosidase. The deleterious effects of introducing these substitutions into the cDNA for acid $\beta$-glucosidase on the catalytic turnover rate of the expressed proteins demonstrate causality of these mutations for GD. As these and earlier (15-17) studies demonstrate, there is, in general, good correlation between the properties of the mutant proteins overexpressed in $\mathrm{Sf} 9$ cells and the severity of the disease manifestations in Gaucher patients. This Sf9 expression system permits the overexpression of sufficient protein for further assessment of defective enzyme function, such as binding affinities, thermal stability, $\mathrm{pH}$ sensitivity, and activation by phosphatidylserine or saposin $\mathrm{C}$ (17). Our findings extend the earlier assignments of functional domains of acid $\beta$-glucosidase (17), although more definite identification of residues comprising the active site, as well as activator binding sites, awaits a crystal structure.

For the R48W mutant, the nonconservative substitution of tryptophan for arginine at residue 48 involves the loss of a positive charge and the introduction of a more sterically rigid, bulky side chain, and thus could be expected to be a very deleterious defect. However, the normal stability and interaction of the R48W mutant enzyme with active site-directed inhibitors argued against a gross structural distortion of the expressed protein, in particular at the catalytic site, and suggested that residue 48 may lie near the surface of the protein or in a location where such a substitution could be better accommodated. To date, characterization of the $\mathrm{NH}_{2}$-terminal portion of human acid $\beta$-glucosidase has been limited. Only three other patient-derived single missense mutations (T43I, reference 34, and V15L and G46E, reference 40) have been reported in the first 100 amino acids of the human enzyme, but only the very conservative V15L mutant has been expressed (40) and shown to retain significant residual activity $(\sim 14 \%$ that of the normal). Earlier mutagenesis studies in this laboratory (15) involving the engineered (i.e., not patient-derived) mutation T43K strongly supported the hypothesis that the region encompassing this amino acid is not buried within the protein core, since introduction of this highly unfavorable substitution (polar, uncharged threonine for the large positively charged lysine) had no effect on activity. Additionally, the presence of oligosaccharide chains attached at $\mathrm{Asn}^{19}$ and $\mathrm{Asn}^{59}$ (proven sites of N-glycosylation; reference 41) would be expected to bring these residues and the surrounding regions to the surface.

In the S271N allele, serine is replaced by the bulkier asparagine, which also has an uncharged polar R group. Studies have shown that this substitution is generally nondisruptive (42), unless buried inside the protein in a region of very tight packing. Since $\mathrm{Asn}^{270}$ has been shown to be one of four sites of $\mathrm{N}$-glycosylation in acid $\beta$-glucosidase (41), the S271N lesion should be near the surface. Although only the presence of a proline in the second position of the consensus sequence for $\mathrm{N}$-glycosylation (Asn-Xaa-Ser/Thr, where Xaa $\neq$ Pro) prevents attachment of oligosaccharide chains (43), the severe reduction in activity in the S271N mutant (normal sequence, Asn-Ser271_Thr-His; and mutant sequence, Asn-Asn ${ }^{271}$-ThrHis) may be due to interference with the frequency of site occupancy of $\mathrm{Asn}^{270}$.

The reduced activity and altered affinity towards active site-directed inhibitors by the V352L, G202R, and G195E mutant polypeptides localize residues 352, 202, and 195 to the active site of acid $\beta$-glucosidase. Substitution of leucine for valine is generally considered a conservative $(44,45)$ or favorable (42) substitution since both have nonpolar R groups varying only by one methylene group. However, residue 352 lies within the region of acid $\beta$-glucosidase containing the substrate and activator binding domains (residues 323-463) (17) and the catalytic nucleophile $\left(\mathrm{Glu}^{340}\right)$ (18). Compared with that of the normally expressed enzyme, the reduced CRIM SA of the V352L enzyme and its lower affinity for the active site-directed inhibitors (especially DNM and CBE) suggest an altered substrate binding region, presumably accounting for its 15 -fold reduction in catalytic efficiency. With respect to the G202R allele, the substitution of the bulky, polar arginine for the small, nonpolar glycine is considered unfavorable, especially if amino acid position 202 is in a tightly packed region of the folded protein (42). That Gly ${ }^{202}$ contributes to active site structure, either directly by proximity or indirectly via folding, is supported by the decreased affinity of the mutant enzyme for CS, DNM, and $\mathrm{CBE}$, indicating a distorted catalytic site. With the unfavorable substitution of the small, neutral side chain of glycine by the bulky, negatively charged chain of glutamate, the G195E allele had a predictably greatly reduced catalytic efficiency and an altered active site (indicated by the higher $\mathrm{IC}_{50}$ values). $\mathrm{G} 195 \mathrm{E}$ is the first mutation demonstrated to significantly interfere with the binding of CS at the active site. Since mutations at other positions in the active site (e.g., N370S and V394L) selectively interfered with the binding of DNM and CBE, but not significantly with CS (15-17), the side chain of $\mathrm{Glu}^{195}$ in the G195E mutant must have a specific unfavorable interaction with the rigid bicyclic $\mathrm{CS}$ in the aberrant glycopeptide.

Of the numerous residues that have been localized to the active site region of acid $\beta$-glucosidase, only E340 has been assigned a direct mechanistic role (i.e., the catalytic nucleophile) (18). The acid catalyst has yet to be identified. Using hydrophobic cluster analysis to theoretically predict catalytic residues in several lysosomal glycosidases, Henrissat et al. (19) proposed that $\mathrm{Glu}^{235}$ is the acid catalyst in acid $\beta$-glucosidase. The mutagenesis results presented here confirm that $\mathrm{Glu}^{235}$ is required to maintain enzymatic activity, since its substitution by glycine $(\mathrm{E} 235 \mathrm{G})$ resulted in a stable protein with a $>1000$ fold reduction in rate constant. However, a mechanistic role of $\mathrm{Glu}^{235}$ as the acid catalyst could not be confirmed, since no effect on the initial rates of hydrolysis of a panel of substrates with varying leaving group abilities was observed in the presence of a competitive nucleophile (sodium azide). This criterion has been used to assign mechanistic roles to putative acid/ base catalysts in fungal glycosidases (39).

The characteristics of these newly identified mutations in acid $\beta$-glucosidase were generally consistent with the observed phenotypes of the respective types 1 and 2 patients. As expected from the results of large population screening studies $(23,46,47)$ and earlier structure/function studies (15-17), the 
presence of one N370S allele protected against the development of neurological manifestations, even if the heteroallelic mutation was severely compromised, as in patients 4 (N370S/ G195E) and 5 (N370S/1450del2). With a CRIM SA value similar to that of N370S, the R48W mutant also had sufficient residual activity to protect against the neuronopathic forms of GD, as indicated by the type 1 phenotype of Patient 1 (R48W/ W179X), where the truncated W179X does not produce any active acid $\beta$-glucosidase protein. While these studies were in progress, the R48W mutation was identified (but not expressed or characterized) in two GD patients with mild (R48W/?; African-American) and moderate to severe (R48W/ L444P; Bedouin descent) type 1 disease (34).

The type 1 phenotypes of patients 2 and 3 suggest that the V352L and S271N alleles each have sufficient residual activity to prevent neurologic disease, since the heteroalleles in these patients (i.e., a "deletion" and RecNciI, respectively) most likely contribute little, if any, residual activity. The protective effect of S271N would not be predicted from its low CRIM SA ( $\sim 0.02$, average of four experiments), illustrating the limits of an in vitro detergent-based assay (which may not always reflect a mutant's intracellular activity at the lysosomal level) or that other factors (such as a modifier gene) in addition to acid $\beta$-glucosidase deficiency may influence the phenotypic expression of GD. The role of a modifier gene is clearly indicated by the range of phenotypes (asymptomatic to mild to severe disease) observed in Gaucher type 1 patients who are homoallelic for N370S.

The homozygous presence of the severely compromised G202R mutant allele, with its 100 -fold reduction in turnover rate, is in agreement with the type 2 phenotype of patient 7 and with the findings of Beutler et al. (35), who identified the G202R mutation in two GD patients with the least severe type 1 phenotype (G202R/N370S) and in one type 2 patient $(\mathrm{G} 202 \mathrm{R} / \mathrm{L} 444 \mathrm{P})$. The combination of the inactive truncation allele, K74X, and the severely compromised F213I allele (36) also is consistent with the observed type 2 phenotype of patient 6. Interestingly, the F213I allele has been identified in all three types of GD $(22,36)$, including an English type 1 patient whose heteroallele was the severely deficient P289L allele (22), which again suggests a role for a modifier gene in the phenotypic expression of this disease.

\section{Acknowledgments}

The authors thank Xiao Lan Zhang, M.S. and Tamar Erlich, M.S. for excellent technical help, and Ms. Elizabeth Miller and Ms. Amy Piatek for help with the mutagenesis and characterization studies.

This work was supported in part by a grant (6-FY95-0382) from the March of Dimes Birth Defects Foundation (M.E. Grace), a grant (5 MO1 RR0071) for the Mount Sinai General Clinical Research Center from the National Center for Research Resources, National Institutes of Health (NIH), and an NIH grant (5 P30 HD28822) for the Mount Sinai Child Health Research Center. G.M. Pastores was the recipient of a Young Pediatric Investigator Award and currently is the recipient of a Minority Clinical Associate Physician Award (5M01-RR0071) from the National Center for Research Resources of the NIH.

\section{References}

1. Beutler, E., and G.A. Grabowski. 1994. Gaucher disease. In The Metabolic Basis of Inherited Disease. C.R. Scriver, A.L. Beaudet, W.S. Sly, and D.
Valle, editors. McGraw Hill Inc., New York. 2641-2670.

2. Desnick, R.J., S. Gatt, and G.A. Grabowski. 1982. Gaucher Disease: A Century of Delineation and Research. Alan R. Liss, Inc., New York.

3. Devine, E.A., M. Smith, F.X. Arredondo-Vega, B. Shafit-Zagardo, and R.J. Desnick. 1982. Chromosomal localization of the gene for Gaucher disease. Prog. Clin. Biol. Res. 95:511-534.

4. Barneveld, R.A., W. Keijzer, F.P.W. Tegelaers, E.I. Ginns, A. Geurtsvan Kessel, R.O. Brady, J.A. Barranger, J.M. Tager, H. Galjaard, A. Westerveld, and A.J.J. Reuser. 1983. Assignment of the gene coding for human beta-glucocerebrosidase to the region $\mathrm{q} 21-\mathrm{q} 31$ of chromosome 1 monoclonal antibodies. Hum. Genet. 64:227-231.

5. Ginns, E.I. 1985. Gene mapping and leader polypeptide sequence of human glucocerebrosidase: implications for Gaucher disease. Proc. Natl. Acad. Sci. USA. 82:7101-7105.

6. Beutler, E., and T. Gelbart. 1997. Hematologically important mutations: Gaucher disease. Blood Cells Mol. Dis. 23:2-7.

7. Beutler, E. 1992. Gaucher disease: new molecular approaches to diagnosis and treatment. Science (Wash. DC). 256:794-799.

8. Horowitz, M., and A. Zimran. 1994. Mutations causing Gaucher disease. Hum. Mutat. 3:1-11.

9. Beutler, E. 1993. Gaucher disease as a paradigm of current issues regarding single gene mutations of humans. Proc. Natl. Acad. Sci. USA. 90:5384-5390.

10. Sorge, J., C. West, B. Westwood, and E. Beutler. 1985. Molecular cloning and nucleotide sequence of human glucocerebrosidase cDNA [published erratum appears in Proc. Natl. Acad. Sci. USA. 1986 83:3576]. Proc. Natl. Acad. Sci. USA. 82:7289-7293

11. Tsuji, S., P.V. Choudary, B.M. Martin, S. Winfeild, J.A. Barranger, and E.I. Ginns. 1986. Nucleotide sequence of cDNA containing the complete coding sequence for human cerebrosidase. J. Biol. Chem. 261:50-53.

12. Horowitz, M., S. Wilder, Z. Horowitz, O. Reiner, T. Gelbart, and E. Beutler. 1989. The human glucocerebrosidase gene and pseudogene: structure and evolution. Genomics. 4:87-96.

13. Sorge, J., E. Gross, C. West, and E. Beutler. 1990. High level transcription of the glucocerebrosidase pseudogene in normal subjects and patients with Gaucher disease. J. Clin. Invest. 86:1137-1141.

14. Ho, M.W., and J.S. O'Brien. 1971. Gaucher's disease: deficiency of 'acid' $\beta$-glucosidase and reconstitution of enzyme activity in vitro. Proc. Natl. Acad. Sci. USA. 68:2810-2813.

15. Grace, M., P. Graves, F. Smith, and G. Grabowski. 1990. Analyses of catalytic activity and inhibitor binding of human acid $\beta$-glucosidase by sitedirected mutagenesis. J. Biol. Chem. 265:6827-6835.

16. Grace, M., A. Berg, G. He, L. Goldberg, M. Horowitz, and G. Grabowski. 1991. Gaucher disease: heterologous expression of two alleles associated with neuronopathic phenotypes. Am. J. Hum. Genet. 46:646-655.

17. Grace, M.E., K.M. Newman, V. Scheinker, A. Berg-Fussman, and G.A Grabowski. 1994. Analysis of human acid $\beta$-glucosidase by site-directed mutagenesis and heterologous expression. J. Biol. Chem. 269:2283-2291.

18. Miao, S., J.D. McCarter, M.E. Grace, G.A. Grabowski, R. Aebersold, and S.G. Withers. 1994. Identification of $\mathrm{Glu}^{340}$ as the active-site nucleophile in human glucocerebrosidase by use of electrospray tandem mass spectrometry. $J$. Biol. Chem. 269:10975-10978.

19. Henrissat, B., I. Callebaut, S. Fabrega, P. Lehn, J.-P. Mornon, and G. Davies. 1995. Conserved catalytic machinery and the prediction of a common fold for several families of glycosyl hydrolases. Proc. Natl. Acad. Sci. USA. 92: 7090-7094.

20. Sambrook, J., E. Fritsch, and T. Maniatis. 1989. Molecular cloning. Cold Spring Harbor Laboratory Press, Cold Spring Harbor, New York. 9.16-9.23 pp.

21. Beutler, E., and T. Gelbart. 1990. Gaucher disease associated with a unique Kpnl restriction site: identification of the amino acid substitution. Ann. Hum. Genet. 54:149-153.

22. He, G.-S., M.E. Grace, and G.A. Grabowski. 1992. Gaucher disease: four rare alleles encoding F213I, P289L, T323I, and R463C in type 1 variants. Hum. Mutat. 1:423-427.

23. Sibille, A., C. Eng, S.-J. Kim, G. Pastores, and G.A. Grabowski. 1993. Phenotype/genotype correlations in Gaucher disease type 1: clinical and therapeutic implications. Am. J. Hum. Genet. 52:1094-1101.

24. Tsuji, S., P. Choudary, B. Martin, B. Stubblefield, J. Mayor, J. Barranger, and E. Ginns. 1987. A mutation in the human glucocerebrosidase gene in neuronopathic Gaucher's disease. N. Engl. J. Med. 316:570-575.

25. Sanger, F. 1981. Determination of nucleotide sequences in DNA. Science (Wash. DC). 214:1205-1208.

26. Chen, E.Y., and P.H. Seeburg. 1985. Supercoil sequencing: a fast and simple method for sequencing plasmid DNA. DNA (NY). 4:165-170.

27. Sorge, J., C. West, B. Westwood, and E. Beutler. 1986. Correction Proc. Natl. Acad. Sci. USA. 1985. 82:7289-7293. Proc. Natl. Acad. Sci. USA. 83:3567.

28. Beutler, E., C. West, and T. Gelbart. 1992. Polymorphisms in the human glucocerebrosidase gene. Genomics. 12:795-800.

29. Taylor, J.W., J. Ott, and F. Eckstein. 1985. The rapid generation of oligonucleotide-directed mutations at high frequency using phosphorothioatemodified DNA. Nucleic Acids Res. 13:8765-8785.

30. Taylor, J.W., W. Schmidt, R. Cosstick, A. Okruszek, and F. Eckstein 1985. The use of phosphorothioate-modified DNA in restriction enzyme reac- 
tions to prepare nicked DNA. Nucleic Acids Res. 13:8749-8764.

31. O'Reilly, D., L. Miller, and V. Luckow. 1994. Baculovirus expression vectors: a laboratory manual. Oxford University Press, Inc., New York. 1-347 pp.

32. Schagger, H., and G. von Jagow. 1987. Tricine-sodium dodecyl sulfatepolyacrylamide gel electrophoresis for the separation of proteins in the range from 1 to $100 \mathrm{kDa}$. Anal. Biochem. 166:368-379.

33. Dinur, T., G.A. Grabowski, R.J. Desnick, and S. Gatt. 1984. Synthesis of a fluorescent derivative of glucosyl ceramide for the sensitive determination of glucocerebrosidase activity. Anal. Biochem. 136:223-234.

34. Beutler, E., T. Gelbart, A. Demina, A. Zimran, and P. LeCoutre. 1995. Five new Gaucher disease mutations. Blood Cells Mol. Dis. 21:20-24.

35. Beutler, E., A. Demina, and T. Gelbart. 1994. Glucocerebrosidase mutations in Gaucher disease. Mol. Med. 1:82-92.

36. Kawame, H., and Y. Eto. 1991. A new glucocerebrosidase-gene missense mutation responsible for neuronopathic Gaucher disease in Japanese patients [published erratum appears in Am. J. Hum. Genet. 1992. 50:885]. Am. J. Hum. Genet. 49:1378-1380.

37. Holliday, R., and G.W. Grigg. 1993. DNA methylation and mutation. Mutat. Res. 285:61-67.

38. Barker, D., M. Schafer, and R. White. 1984. Restriction sites containing CpG show a higher frequency of polymorphism in human DNA. Cell. 36:131138.

39. Wang, Q., D. Trimbur, R. Graham, R. Warren, and S. Withers. 1995. Identification of the acid/base catalyst in Agrobacterium faecalis $\beta$-glucosidase by kinetic analysis. Biochemistry. 34:14554-14562.

40. Kim, J.-W., B.B. Liou, M.-Y. Lai, E. Ponce, and G.A. Grabowski. 1996 Gaucher disease: identification of three new mutations in the Korean and Chinese (Taiwanese) populations. Hum. Mutat. 7:214-218

41. Berg-Fussman, A., M.E. Grace, Y. Ioannou, and G. Grabowski. 1993. Human acid $\beta$-glucosidase: $\mathrm{N}$-glycosylation site occupancy and the effect of glycosylation on enzymatic activity. J. Biol. Chem. 268:14861-14866.

42. Bordos, D., and P. Argos. 1991. Suggestions for "safe" residue substitutions in site-directed mutagenesis. J. Mol. Biol. 217:721-729.

43. Roitsch, T., and L. Lehle. 1989. Structural requirements for protein N-glycosylation. Influence of acceptor peptides on cotranslational glycosylation of yeast invertase and site-directed mutagenesis around a sequon sequence. Eur. J. Biochem. 181:525-529.

44. Grantham, R. 1974. Amino acid difference formula to help explain protein evolution. Science (Wash. DC). 185:862-864.

45. Hutchinson, C., R. Swanstrom, and D. Loeb. 1991. Complete mutagenesis of protein coding domains. Methods Enzymol. 202:356-390.

46. Zimran, A., J. Sorge, E. Gross, M. Kubitz, C. West, and E. Beutler. 1989. Prediction of severity of Gaucher's disease by identification of mutations at DNA level. Lancet. ii:349-352.

47. Theophilus, B., T. Latham, G.A. Grabowski, and F.I. Smith. 1989. Gaucher's disease: molecular heterogeneity and phenotype correlations. Am. J. Hum. Genet. 45:212-225. 